\title{
Why Simple Shrinkage is Still Relevant for Redundant Representations?
}

\author{
Michael Elad \\ Department of Computer Science - The Technion - Israel Institute of Technology \\ Haifa 32000 Israel, email: elad@cs.technion.ac.il \\ Phone: +972-4-829-4169 Fax: +972-4-829-3900
}

May 29, 2005

\section{General Description}

- Problem statement: Shrinkage is a well known and appealing denoising technique, introduced originally by Donoho and Johnstone in 1994. The use of shrinkage for denoising is known to be optimal for Gaussian white noise, provided that the sparsity on the signal's representation is enforced using a unitary transform. Still, shrinkage is also practiced with non-unitary, and even redundant representations, typically leading to satisfactory results. In this paper we shed some light on this behavior.

- Originality of the work: The main argument in this paper is that such simple shrinkage could be interpreted as the first iteration of an algorithm that solves the basis pursuit denoising (BPDN) problem. While the desired solution of BPDN is hard to obtain in general, a simple iterative procedure that amounts to step-wise shrinkage can be employed with quite successful performance.

- New results: We demonstrate how the simple shrinkage emerges as the first iteration of such algorithm. Furthermore, we show how shrinkage can be iterated, turning into an effective algorithm that minimizes the BPDN via simple shrinkage steps, in order to further strengthen the denoising effect. Lastly, the emerging algorithm stands in between the basis and the matching pursuit as a novel and appealing pursuit technique for atom decomposition in the presence of noise. 


\section{Extended Abstract}

The maximum a-posteriori probability (MAP) approach towards the denoising problem proposes the minimization of the function

$$
f(\mathbf{x})=\frac{1}{2} \cdot\|\mathbf{x}-\mathbf{y}\|_{2}^{2}+\lambda \cdot P_{r}\{\mathbf{x}\} .
$$

The first term is known as the log-likelihood, describing the relation between the desired (clean) signal, $\mathbf{x} \in \mathbb{R}^{N}$, and a noisy version of it $\mathbf{y} \in \mathbb{R}^{N}$. We assume the model $\mathbf{y}=\mathbf{x}+\mathbf{v}$, with $\mathbf{v} \in \mathbb{R}^{N}$ a Gaussian zero mean white noise. The term $P_{r}\{\mathbf{x}\}$ stands for a prior posed on the unknown signal $\mathbf{x}$. Numerous expressions that have been used for various signal types can be found in the literature. Among the popular methods that are now considered as classic methods in signal processing, we mention (i) energy minimization $P_{r}\{\mathbf{x}\}=\|\mathbf{x}\|_{2}^{2}$; (ii) smoothness penalty $P_{r}\{\mathbf{x}\}=\|\mathbf{L x}\|_{2}^{2}$; (iii) total variation $P_{r}\{\mathbf{x}\}=\|\nabla \mathbf{x}\|_{1}$; and (iv) sparsity of the unknown signal with respect to its transformed representation, $P_{r}\{\mathbf{x}\}=\|\mathbf{T x}\|_{1}$.

Formulations of the denoising problem as in (1) are commonly used, and are the basis for many more general inverse problems. Using priors such as TV and other robust methods, the above opens an opportunity to the study of non-linear filtering, typically formulated as partial differential equations (PDE). Those are typically handled by iterative numerical solvers, that are characterized as slow and cumbersome.

In parallel to the progress made in the PDE directions, Donoho and Johnstone pioneered a wavelet based signal denoising algorithm in line with the above list of priors (item \#5). In a sequence of publications they advocated the use of sparsity of the wavelet coefficients as a driving force in recovering the desired signal $[1,2,3,4,5,6]$. Later work simplified these ideas and related them to the MAP formulation as presented above $[7,8]$, using the prior $P_{r}\{\mathbf{x}\}=\|\mathbf{W} \mathbf{x}\|_{p}$, with a unitary wavelet transform matrix $\mathbf{W} \in \mathbb{R}^{N \times N}$, and $0 \leq p \leq 1$.

Interestingly, using such a prior in Equation (1) leads to a simple closed-form solution, known as shrinkage. This solution amounts to a wavelet transform on the noisy signal, followed by a look-up-table (LUT) function on the coefficients (that depends on $p$ ), $\mathcal{S}\{\mathbf{W} \mathbf{y}\}$, and finally, an inverse wavelet transform to produce the outcome $\hat{\mathbf{x}}$. Such a direct solution stands as a refreshing alternative to the iterative and slow methods mentioned above. This shrinkage algorithm becomes indeed the optimal solver of (1). This optimality depends strongly on the $\ell^{2}$-norm used in evaluating the distance $\mathbf{x}-\mathbf{y}$, and this has direct roots in the white Gaussianity assumptions on the noise. Also, crucial to the optimality of this method is the orthogonality of W.

A new trend of recent years is the use of overcomplete transforms, replacing the traditional unitary ones (see $[9,10,11,12,13,14,15,16,17,18,19,20,21,22,23,24,25]$ as representative works). This trend was partly motivated by the growing realization that orthogonal wavelets are weak in describing the singularities found in images. Another driving force in the introduction of redundant representations is the sparsity in the representation it can provide, which many applications find desirable. Finally, we should mention the desire to obtain shift-invariant transforms, again calling for redundancy in the representation.

In these methods the transform is defined via a non-square full rank matrix $\mathbf{T} \in \mathbb{R}^{L \times N}$, with $L>N$. Such redundant methods, like the un-decimated wavelet transform, curvelet, contourlet, steerable-wavelet, and more, were shown to be more effective in representing images, and other signal types. It is often assumed that $\mathbf{T}$ is tight frame, implying that $\alpha \mathbf{T}^{T} \mathbf{T}=\mathbf{I}$. In such a case, the adjoint $\mathbf{T}^{T}$ stands for the inverse transform, up to the constant $\alpha$.

Given a noisy signal $\mathbf{y}$, we can still follow the shrinkage procedure, by computing the forward transform $\mathbf{T y}$, putting the coefficients through a shrinkage LUT operation $\mathcal{S}\{\mathbf{T y}\}$, and finally applying the inverse transform ${ }^{1}$ to obtain the denoised outcome, $\mathbf{T}^{+} \mathcal{S}\{\mathbf{T y}\}$. Will this be the solver of (1) when using the prior $P_{r}\{\mathbf{x}\}=\|\mathbf{T} \mathbf{x}\|_{p}$ ? The answer is no! As we have said before, the orthogonality of the transform plays a

\footnotetext{
${ }^{1}$ There are infinitely many ways to define the inverse, and in most cases the Moore-Penrose pseudo-inverse is practiced.
} 
crucial role in the construction of the shrinkage as an optimal procedure. Still, shrinkage is practiced quite often with non-unitary, and even redundant representations, typically leading to satisfactory results (see $[10,11,12,13,14,15,16,17,18,19,20]$ as representative examples). Naturally, we should wonder why this is so.

In this work we shed some light on this behavior. Our main argument is that such a shrinkage could be interpreted as the first iteration of a converging algorithm that solves the basis pursuit denoising (BPDN) problem. The BPDN forms a similar problem to the one posed in (1), replacing the analysis prior with a generative one. While the desired solution of BPDN is hard to obtain in general, a simple iterative procedure that amounts to step-wise shrinkage can be employed with quite successful performance. Thus, beyond showing that shrinkage has justified roots in solid denoising methodology, we show how shrinkage can be iterated in a simple form, to further strengthen the denoising effect. Finally, a consequence of the above claims is that sequential application of shrinkage in the way described in this work could be interpreted as a new pursuit algorithm that has similarity to both the matching and the basis pursuit $[26,27,28]$, and yet it is different from both. Such an algorithm can be deployed to seek the sparsest representation of signals, using only simple inner-products of the overall dictionary by the signal (or its residual), and simple shrinkage operations.

\section{References}

[1] Donoho, D.L and Johnstone, I.M. (1994) Ideal spatial adaptation by wavelet shrinkage, Biometrika Vol. 81 No. 3, pp. 425-455, September.

[2] Donoho, D.L. (1995) De-noising by soft thresholding, IEEE Transactions on Information Theory, Vol. 41, No. 3, pp. 613-627, May.

[3] Donoho, D.L., Johnstone, I.M., Kerkyacharian, G., and Picard, D (1995) Wavelet shrinkage - asymptopia, Journal Of The Royal Statistical Society Series B - Methodological, Vol. 57, No. 2, pp. 301-337.

[4] Donoho, D.L. and Johnstone, I.M. (1995) Adapting to unknown smoothness via wavelet shrinkage, Journal of the American Statistical Association, Vol. 90 No. 432, pp. 1200-1224, December.

[5] Donoho, D.L. (1998) Wedgelets: Nearly minimax estimation of edges, Annals Of Statistics, Vol. 27, No. 3, pp. 859-897, June.

[6] Donoho, D.L. and Johnstone, I.M. (1998) Minimax estimation via wavelet shrinkage, Annals of Statistics, Vol. 26, No. 3, pp. 879-921, June.

[7] Moulin, P. and Liu, J. (1999) Analysis of multiresolution image denoising schemes using generalized Gaussian and complexity priors, IEEE Transactions on Information Theory, Vol. 45, No. 3, pp. 909-919, April.

[8] Jansen, M. (2001) Noise Reduction by Wavelet Thresholding, SpringerVerlag, New York.

[9] Candes, E.J. and Donoho, D.L. (2002) Recovering edges in ill-posed inverse problems: optimality of curvelet frames, Annals of Statistics, Vol. 30, No. 3, pp. 784-842, June.

[10] Starck, J.-L, Candes, E.J, and Donoho, D.L. (2002) The curvelet transform for image denoising, IEEE Transactions On Image Processing, Vol. 11, No. 6, pp. 670-684, June.

[11] Starck, J.-L., Elad, M., and Donoho D.L. (2004) Redundant multiscale transforms and their application for morphological component separation, Advances in Imaging And Electron Physics, Vol. 132, pp. 287-348. 
[12] Do, M.N. and Vetterli, M. (2002) Rotation invariant texture characterization and retrieval using steerable wavelet-domain hidden markov models, IEEE Transactions On Multimedia Vol. 4, No. 4, pp. 517-527, December.

[13] Do, M.N. and Vetterli, M. (2003) Framing pyramids, em IEEE Transactions On Signal Processing, Vol. 51, No. 9, pp. 2329-2342, September.

[14] Ishwar, P. and Moulin, P. (2000) Shift invariant restoration - an overcomplete maxent MAP framework, Proceedings of the International Conference on Image Processing, Vol. 3, pp. 270-272.

[15] Do, M.N. and Vetterli, M. (2003) The finite ridgelet transform for image representation, em IEEE Transactions On Image Processing, Vol. 12, No. 1, pp. 16-28 January.

[16] Carre, P. and Andres, E. (2004) Discrete analytical ridgelet transform, Signal Processing, Vol. 84, No. 11, pp. 2165-2173, November.

[17] Lang, M., Guo, H., and Odegard, J.E. (1996) Noise reduction using undecimated discrete wavelet transform, IEEE Signal Processing Letters, Vol. 3, No. 1, pp. 10-12, January.

[18] Chandrika, K., Fodor, I.K., and Gyaourova, A. (2002) Undecimated wavelet transforms for image denoising, Lawrence Livermore National Laboratory, technical report UCRL-ID-150931, November.

[19] Eslami, R. and Radha, H. (2003) The contourlet transform for image de-noising using cycle spinning, in Proceedings of Asilomar Conference on Signals, Systems, and Computers, pp. 1982-1986, November.

[20] Eslami, R. and Radha, H. (2004) Translation-invariant contourlet transform and its application to image denoising, submitted to the IEEE Transactions on Image Processing, October.

[21] Scharcanski, J., Jung, C.R., and Clarke, R.T. (2002) Adaptive image denoising using scale and space consistency, IEEE Transactions On Image Processing, Vol. 11, No. 9, pp. 1092-1101, September.

[22] Jung, C.R. and Scharcanski, J. (2003) Adaptive image denoising and edge enhancement in scale-space using the wavelet transform, Pattern Recognition Letters, Vol. 24, No. 7, pp. 965-971, April.

[23] Portilla, J., Strela, V., Wainwright, M.J, and Simoncelli, E.P. (2001) Adaptive Wiener denoising using a Gaussian scale mixture model in the wavelet domain, Proceedings of the 8th International Conference of Image Processing, Thessaloniki, Greece. October.

[24] Portilla, J., Strela, V., Wainwright, M.J, and Simoncelli, E.P. (2003) Image denoising using scale mixtures of gaussians in the wavelet domain IEEE Transactions On Image Processing, Vol. 12, No. 11, pp. 1338-1351, November.

[25] Guleryuz, O.G. (2003) Weighted overcomplete denoising, Proceedings of the Asilomar Conference on Signals and Systems, Pacific Grove, CA, November.

[26] Mallat, S. \& Zhang, Z. (1993) Matching Pursuit in a time-frequency dictionary, IEEE Transactions on Signal Processing, Volume 41, pages 3397-3415.

[27] Pati, Y.C., Rezaiifar, R. \& Krishnaprasad, P.S. (1993) Orthogonal Matching Pursuit: Recursive Function Approximation with Applications to Wavelet Decomposition, Proceedings of the 27 th Annual Asilomar Conference on Signals, Systems, and Computers.

[28] Chen, S.S., Donoho, D.L. \& Saunders, M.A. (2001) Atomic decomposition by basis pursuit, SIAM Review, Volume 43, number 1, pages 129-59. 